\section{Saúde e Bem-estar na Obesidade: Paradoxo ou Possibilidade?}

\author{
Health and Wellness in Obesity: Paradox or Possibility?
}

\section{RESUMO}

O presente artigo relata, brevemente, a história de um curso comunitário destinado a oferecer intervenções não farmacológicas a mulheres com excesso de peso. Ênfase é dada ao formato atual do curso, que conta com equipe multidisciplinar na área de Saúde apta a oferecer suporte amplo à mulher obesa que encontra dificuldade para se enquadrar o padrão de beleza requerido pela sociedade. Em nosso curso, propomos um programa centrado na saúde e na qualidade de vida do indivíduo obeso, sem foco na perda de peso, de acordo com os princípios da filosofia Health at Every Size. Os resultados qualitativos preliminares indicam que tal abordagem é promissora, oferecendo aos profissionais de saúde uma alternativa à proposta vigente de "perda de peso a qualquer custo". Os avanços no ensino, na pesquisa e na extensão associados ao curso também são discutidos ao longo do artigo.

Palavras-chave: Sobrepeso. Exercício. Nutrição. Filosofia. Qualidade de Vida.

\section{ABSTRACT}

The present manuscript briefly reports on the history of a community program aimed to provide non-pharmacological interventions to overweight women. Focus is given to the current format of the program, which presents a multidisciplinary health team able to providing full support to the obese woman who faces trouble to find the society's beauty standards. In our program, we propose a health and quality of life-oriented approach, without a focus on weight loss, following the philosophical principles from Health at Every Size. The quantitative and qualitative findings reveal that such approach is promising and may provide an alternative mode of intervention to the traditional one, which is focused on the concept of "weight loss at any cost". The advances on teaching, research and community services will be also addressed throughout the manuscript.

\section{Mariana D. Ulian e \\ FERNANDA B. \\ SCAGLIUS I}

Universidade de São Paulo. Faculdade de Saúde Pública, São Paulo, Brasil

Priscila de Morais SATO

Universidade Federal de São Paulo. Instituto de Saúde e Sociedade, São Paulo, Brasil

\section{Bruno T. Modesto, \\ Fabiana B. Benatti e Bruno Gualano}

Universidade de São Paulo. Escola de Educação Física e Esporte, São Paulo, Brasil

\section{Odilon J. Roble}

Universidade de Campinas. Faculdade de Educação Física, São Paulo, Brasil

\section{Ramiro Fernandez UNSAIN}

Universidad Nacional de Buenos Aires. Facultad de Filosofía y Letras, Buenos Aires, Argentina 
Keywords: Overweigh. Exercise. Nutrition. Philosophy. Quality of Life.

\section{INTRODUÇÃO}

\section{Há aproximadamente duas décadas, a Escola de Educação Física e Esporte} da Universidade de São Paulo (EEFE-USP) abriga um tradicional curso comunitário destinado a oferecer atendimento nutricional e prescrição de exercícios a indivíduos obesos. Tal curso foi criado pelo professor Antonio Herbert Lancha Junior em 1995, sendo originalmente denominado Atividade física e controle alimentar para pessoas obesas. O programa tinha como foco a perda de peso corporal por meio de dietas restritivas - prescritas por nutricionistas da equipe - e treinamento físico supervisionado, realizado nas dependências da EEFE-USP.

O curso comunitário sempre serviu bem o propósito de aliar o ensino e a pesquisa à extensão universitária. Ao longo dos anos, foram diversos alunos de graduação e pós-graduação que se valeram da estrutura do curso para a coleta de dados e produção científica. Em 2011, o professor Bruno Gualano assumiu a coordenação do curso e, ao lado do professor Lancha Junior, iniciou uma necessária e profunda repaginação dos serviços prestados. A intenção era oferecer às participantes um maior período de acompanhamento, já que, findo o curso semestral, não havia possibilidade de renovação de matrícula, forçando-as a buscarem - frequentemente sem sucesso - outros centros que oferecessem atendimento similar. Sendo assim, a primeira providência foi ampliar o período de seguimento para, no mínimo, um ano, com possibilidade de renovação adicional em caso de necessidade e de vagas remanescentes.

Em segundo lugar, de modo a usufruir cientificamente do novo modelo do curso, planejou-se estudos de longo prazo a fim de avaliar os efeitos crônicos da intervenção proposta. No entanto, a ineficácia das dietas restritivas em longo prazo é bem documentada na literatura, pois possui baixa aderência e, frequentemente, leva à perda excessiva de massa magra e a recuperação do peso perdido em $95 \%$ dos casos $[1,4$, 6]. Além disso, tais dietas - e, em especial, o inexorável fracasso em segui-las por um longo período - são associados com o sentimento de culpa, sintomas depressivos, insatisfação com o corpo, compulsões alimentares e transtornos alimentares. De fato, a equipe sofreu grande influência de uma filosofia conhecida como Health at Every Size, segundo a qual o peso saudável é definido como aquele que o indivíduo estabelece ao longo de sua vida ao ter um estilo de vida mais satisfatório [2, 4]. Essa filosofia tem como objetivo encorajar comportamentos saudáveis para pessoas de todos os "tamanhos” corporais, sendo a perda de peso uma consequência ou não desse processo. A filosofia Health at Every Size é pautada por cinco princípios, a saber: 1) reconhecer que a saúde e bem-estar são multidimensionais e incluem aspectos emocionais, físicos, espirituais e intelectuais; 2) encorajar a construção de uma imagem corporal positiva; 3 ) aceitar e respeitar a diversidade de tamanhos e formatos corporais; 4) promover uma alimentação que equilibre necessidades nutricionais individuais, além de aspectos como fome, saciedade, apetite e prazer e; 5) promover atividades físicas prazerosas e sustentáveis [7]. 
Atraídos por essa linha de atuação, o curso foi reformulado de maneira a oferecer ao aluno um programa que escapasse do paradigma da "perda de peso a qualquer custo", e, em vez disso, visasse melhorar a saúde geral e a qualidade de vida do participante. Para tanto, passou-se a adotar uma intervenção pautada nos moldes da Clínica Ampliada e Compartilhada [3]. A partir desse modelo, é possível depreender que diferentes abordagens profissionais se complementam, permitindo que distintos enfoques, disciplinas e instrumentos sejam incluídos e articulados. Visa-se, pois, à compreensão ampliada do processo saúde-doença em oposição a intervenções pontuais e isoladas; além disso, procura-se promover autonomia e protagonismo dos sujeitos participantes. A Clínica Ampliada e Compartilhada encoraja o estabelecimento de vínculo e entendimento do contexto de vida do participante e, dessa forma, demanda flexibilidade dos profissionais envolvidos [3].

Em 2013, conduziu-se a primeira versão revisada do curso comunitário. Objetiva-se, com este artigo, discorrer sobre a nova metodologia científica e os princípios filosóficos norteadores desenvolvidos para o programa de intervenção, bem como destacar os resultados qualitativos preliminares obtidos, com ênfase nos avanços detectáveis nos âmbitos do ensino, da pesquisa e da extensão.

\section{MÉTODOS}

Do ponto de vista científico, trata-se de uma intervenção longitudinal, prospectiva e quasi-experimental. Foram selecionadas 30 mulheres com idade entre 25 e 50 anos e com um Índice de Massa Corporal (IMC) acima de $30 \mathrm{~kg} / \mathrm{m}^{2}$. Outros critérios de inclusão foram os seguintes: não ter diabetes mellitus 1 e 2, não realizar acompanhamento nutricional fora da intervenção e não usar remédios para emagrecimento. As características antropométricas das participantes são ilustradas na Tabela 1. O curso teve início em agosto de 2012 e terminou em agosto de 2013. Das trinta participantes selecionadas inicialmente, 14 concluíram a intervenção. As desistências foram motivadas por razões pessoais.

Tabela 1 - Características antropométricas de 14 mulheres obesas que participaram do estudo.

\begin{tabular}{lccc}
\hline VARIÁVEL & MÉdIA & $\begin{array}{c}\text { DESVIO } \\
\text { PADRÃO }\end{array}$ & $\begin{array}{l}\text { VALORES MíNIMOS } \\
\text { E MÁXIMOS }\end{array}$ \\
\hline Idade (anos) & 40,6 & 7,1 & $30-49$ \\
\hline $\begin{array}{l}\text { Peso corporal }(\mathrm{kg}) \\
\text { Índice de massa }\end{array}$ & 97,0 & 16,4 & $75,3-139$ \\
corporal $\left(\mathrm{kg} / \mathrm{m}^{2}\right)$ & 37,1 & & $30,9-47,4$ \\
\hline
\end{tabular}




\begin{tabular}{lccc}
$\begin{array}{l}\text { Circunferência da } \\
\text { cintura }(\mathrm{cm})\end{array}$ & 112,9 & 12,3 & $93,7-133,5$ \\
\hline $\begin{array}{l}\text { Circunferência do } \\
\text { quadril }(\mathrm{cm})\end{array}$ & 127,4 & 14,6 & $107-149$ \\
\hline Massa gorda* (kg) & 43,5 & 4,6 & $36,5-50,5$ \\
\hline & 42,1 & 11,0 & $29,8-67,5$ \\
\hline Massa gorda* (\%) & 7,0 & $45,4-72,4$ \\
\hline
\end{tabular}

* Avaliadas pelo método de pesagem hidrostática.

As participantes realizaram atividade física três vezes por semana e atendimento nutricional individual quinzenalmente. Além disso, participaram de cinco oficinas filosóficas ao longo da intervenção. As atividades físicas incluíram exercícios aeróbicos sistematizados, atividades lúdicas, jogos esportivos e treinamento de força. As aulas foram ministradas por um professor-coordenador e por alunos de graduação da EEFE-USP, na qualidade de monitores do curso. As oficinas filosóficas empregaram conceitos básicos de filosofia e estimularam as participantes a refletirem sobre o tema do desejo e como conduzi-lo, permitindo que construíssem reflexões próprias sobre tais questões. A intervenção nutricional se baseou no aconselhamento nutricional, que consiste num processo de suporte que auxilia o indivíduo a resolver dificuldades alimentares e potencializar seus recursos pessoais por meio de estratégias individualizadas que estimulem a responsabilidade para o autocuidado, sem a prescrição de dietas [8].

A intervenção foi oferecida pela EEFE-USP e contou com a participação de uma equipe multiprofissional (professores de educação física, nutricionistas, médico, antropólogo e filósofo). A equipe ancorou-se no princípio de que é possível manter-se "saudável" e apresentar boa qualidade de vida a despeito do excesso de peso corporal. A fim de testar essa possibilidade, lançou-se mão de métodos de pesquisa qualitativos e quantitativos (modelo misto de pesquisa).

As informações qualitativas foram coletadas por meio de três grupos focais quadrimensais, os quais foram conduzidos por um moderador experiente e um observador. Em tais discussões, abordavam-se questões pertinentes a todas as áreas envolvidas na intervenção, com auxílio de um guia de perguntas elaborado previamente pela equipe multiprofissional. Os grupos focais permitiram observar a evolução das participantes e estabelecer semelhanças e diferenças entre os diferentes momentos registrados. Além disso, forneceram subsídios capazes de nortear mudança nas estratégias utilizadas pelos profissionais, caso pertinente. Todos os grupos focais foram gravados e transcritos para posterior análise.

A cada três meses, realizaram-se avaliações antropométricas, exames de sangue e 
o preenchimento de escalas e questionários autoaplicados para avaliar imagem corporal, atitudes corporais, compulsão alimentar, restrição alimentar e atitudes alimentares transtornadas. Neste artigo, reportamos apenas os achados qualitativos preliminares, obtidos a partir de grupos focais organizados ao longo da intervenção, uma vez que os dados quantitativos permanecem sob análise.

O presente projeto possui aprovação do Comitê de Ética e Pesquisa local e as participantes do curso assinaram um termo de consentimento livre e esclarecido autorizando a divulgação dos resultados.

\section{RESULTADOS}

Há indícios de que o projeto tem atingido seus objetivos, conforme se depreende dos trechos a seguir, transcritos, com autorização das participantes (representadas por nomes fictícios com o intuito de preservar o direto ao anonimato), a partir de análises de grupos focais, conduzidos por um antropólogo (Ramiro). Em itálico, constam os apontamentos feitos por aluna de pós-graduação que atuou como observadora do grupo focal.

\section{Sobre a Atividade Física}

"Maria - Quanto ao fato dos preparadores físicos, realmente a atitude deles para conosco é realmente algo que eu admiro muito. É... Vou contar uma coisa aqui pra vocês agora que vocês vão dizer que é loucura, mas é verdade. Eu trabalho em um CEU da prefeitura de São Paulo, e lá tem todo o tipo de ginástica, tem ioga, tem hidroginástica e eu não tenho coragem de fazer nada. Por quê? Porque eu comecei a fazer ginástica e a professora ela ficava fazendo comentários do tipo é... [engasga... começa a tentar não chorar].

Ramiro - O que falavam, Maria? Mas falavam de você? Comentários de você?

Maria - Que eu não conseguia alcançar, que eu era muito lenta...

[Ramiro descontrai o grupo, xinga a moça que falava que a Maria era lenta (risos de todas)].

Maria - E ela é minha colega de trabalho.

Ângela - É uma infeliz!

Ramiro - É uma infeliz, muito bem, Ângela!

Maria - Meio que o trauma que eu já tenho disso aumentou entendeu? (...) Porque aqui ninguém me conhecia, aqui ninguém... Entendeu? Exatamente por isso, que eu viajo pra chegar até aqui, pra não correr esse risco. [Maria fala que aqui se sente à vontade para fazer ginástica - segurando o choro - Joana faz um carinho no antebraço de Maria].”

\section{Sobre a Oficina Filosófica e o Aconselhamento Nutricional}

"Ramiro (...) Se pensássemos em termos de ferramentas. Antes de começar todo esse programa e agora, que ferramentas vocês têm? Melhores, piores? Que elementos vocês conseguem utilizar para lidar com o assunto do peso?

Katia - Ah, de que é... Eu aprendi muita coisa assim, de que você não precisa cortar as coisas que 
você gosta, você tem só que diminuir... Né, tentar pelo menos, porque é difícil, porque uma coisa que você gosta né, quer aproveitar bastante, mas é... Ter um autocontrole, tentar ter né, tentar ter, mas não de deixar de comer aquilo que a gente gosta.

Paula - É impossível né.

Katia - Eu já fiz muito isso, hoje eu não faço mais.

Paula - [Fala com muita firmeza e tranquilidade sobre não deixar de comer doces, já que ela gosta] (...) Você muda alguns hábitos. Doce é uma coisa que eu sempre gostei e não vou deixar de comer, mas também não vou ficar comprando em quantidade né. A questão do Odilon (filósofo da equipe) te faz refletir muito. A palestra dele não é só aqui, terminou a palestra... Você fica refletindo. Que nem, na última que teve, uma última que teve que eu não vim, ela comentou aquela coisa do trânsito...

Débora - Do querer, do querer...

Paula - E eu achei o máximo isso. Aí você fica pensando a semana toda.

Débora - Que nem tudo que você quer você vai e faz. Por exemplo, você tá no trânsito o cara vai lá e te fecha, você vai querer matar ele, você não vai matar. A mesma coisa com a comida: se tá ali você não precisa comer tudo, ou coisa parecida. A vontade você tem, mas você não precisa daquilo, passa entendeu, é uma vontade que passa, é uma raiva que passa, tudo passa né... Então aí você tem que saber controlar.

Paula - Então na realidade você vai fazendo seu autocontrole né... Seu autocontrole. Porque não adianta fazer só o programa e depois largar tudo né. Isso aqui na verdade você tem que incorporar.

Katia - É, não adianta ficar só por esse um ano. Aí faz a gente pensar que não é só pra agora...

Paula - Não, não é só pra agora...

Katia - É uma coisa que você tem que levar pra vida.

Débora - É pra vida.”

\section{DISCUSSÃO}

Este artigo traz os resultados preliminares obtidos na primeira edição atualizada de um curso comunitário que tem como objetivo central proporcionar saúde e qualidade de vida à mulher obesa, sem, contudo, focar na perda de peso corporal.

Os trechos extraídos dos grupos focais destacados neste artigo sugerem que as participantes melhoraram o comportamento alimentar e sentiram-se acolhidas e confortáveis para praticar atividades físicas, bem como para discutir aspectos relacionados ao corpo e ao peso corporal. Além disso, as participantes reportaram que o curso foi uma experiência enriquecedora e pareceram confiantes e seguras para dar seguimento ao que aprenderam durante a intervenção.

É premente salientar que a discutida remodelação do curso - atualmente divulgado à comunidade como Saúde e Bem-estar na Obesidade -, só se fez possível graças à inestimável participação de pesquisadores com formações e interesses de pesquisa diversificados e complementares que aderiram ao projeto. Além disso, faz-se necessário destacar que o curso comunitário conta, atualmente, com a contribuição de alunos de graduação (monitores), mestrado, doutorado e pós-doutorado, além de docentes de quatro diferentes Instituições (Universidade de São Paulo, Universidade Federal 
de São Paulo, Universidade de Campinas e Universidade de Buenos Aires), nas áreas de Educação Física, Nutrição, Filosofia e Antropologia. O apoio institucional também merece destaque; em 2012 e 2013, o curso foi contemplado pela Pró-Reitoria de Cultura e Extensão Universitária com sete bolsas para alunos de graduação, oriundas do programa Aprender com Cultura e Extensão.

Não obstante o fato de que os dados finais ainda estejam sob análise, os excertos obtidos dos grupos focais permitem sugerir que o curso tem produzido uma sensível mudança na forma de pensar o corpo, a alimentação e a prática de exercício. Muitas das participantes, claramente, relegam a perda de peso para segundo plano e comemoram o fato de sentirem-se saudáveis, mesmo que obesas. Decerto, a combinação dos dados qualitativos aos quantitativos fornecerão conclusões robustas acerca da eficácia do curso, possivelmente redundado em bons resultados no âmbito da pesquisa. Ademais, cumpre destacar que compartilhamento horizontal de saberes oportunizado pelo curso possui significado pedagógico ímpar aos alunos de graduação, que ganham elementos importantes para a prática profissional.

\section{CONCLUSÃO}

Os achados qualitativos preliminares descritos neste artigo revelam que a nova metodologia desenvolvida para o programa - com destaque à dieta não prescritiva, a compreensão ampliada do processo saúde-doença e a promoção da autonomia e protagonismo dos participantes com articulação multiprofissional - possui potencial para se tornar uma alternativa plausível capaz de promover saúde, em lato sensu, à mulher com excesso de peso.

Além disso, o curso tem oferecido ao aluno de graduação possibilidade de engajar-se em práticas profissionais multidisciplinares de cuidado à saúde na obesidade, que questionam o tratamento medicalizado da mesma. Por se tratar de uma intervenção plural, o aluno beneficia-se de técnicas de intervenção que serão de grande valia para a formação profissional, oferecendo-lhes oportunidade de sedimentar, na prática e de forma supervisionada, o conhecimento teórico difundido em seus cursos de graduação. Destaca-se o envolvimento destes estudantes com o trabalho interdisciplinar e interprofissional em saúde, algo essencial tendo em vista a complexidade da obesidade como objeto epistêmico e como desafio clínico e de saúde pública. Salienta-se também a exposição destes alunos às chamadas tecnologias leves [5], que são mais aconselhativas e relacionais e se centram no interesse, na escuta, no vínculo e no acolhimento. Tais tecnologias se mostram fundamentais para o trabalho humanizado em Saúde. Esforços permanecem direcionados à investigação científica acerca da exequibilidade, eficácia e segurança do programa proposto no trato da mulher com excesso de peso corporal. 


\section{AGRADECIMENTOS}

Os autores são gratos a todos os alunos de graduação e pós-gradução, técnicos e funcionários que trabalharam ao longo dos anos no curso comunitário. Em especial, faz-se menção à Professora Fernanda Scagliusi - docente da Faculdade de Saúde Pública da Universidade de São Paulo -, responsável por coordenar as ações nutricionais. Além disso, destacam-se as participações do Professor Odilon Roble - docente da Universidade de Campinas -, responsável pelas oficinas filosóficas e do Professor Ramiro Fernandez Unsain - docente da Universidad Nacional de Buenos Aires -, responsável por conduzir os grupos focais. Além disso, os autores agradecem ao apoio da Comissão de Cultura e Extensão da EEFE-USP e à Pró-Reitoria de Cultura e Extensão Universitária da Universidade de São Paulo, pelo apoio corriqueiro.

\section{REFERÊNCIAS}

[1] ABETE, I.; et al. Obesity and metabolic syndrome: Potential benefit from specific nutritional components. Nutrition, Metabolism \& Cardiovascular Diseases. v. 21, n. 2, pp. 1-15, 2011.

[2] BACON, L.; et al. Size acceptance and intuitive eating improve health for obese, female chronic dieters. Journal of the American Dietetic Association. v. 105, n. 6, pp. 929-936, 2005 .

[3] BRASIL. MINISTÉRIO DA SAÚDE. Secretaria de Atenção à Saúde. Clínica ampliada e compartilhada. Brasília, DF: Ministério da Saúde, p. 64, 2009.

[4] GAGNON-GIROUARD, M.P.; et al. Psychological impact of a "Health-at-Every-Size" intervention on weight-preoccupied overweight/obese women. Journal of Obesity. pp. 1-12, 2010.

[5] MERHY, E.E.; FEUERWERKER, L.C.M. Novo olhar sobre as tecnologias de saúde: Uma necessidade contemporânea. In: MANDARINO, A.C.S.; GOMBERG, E. (Org.). Leituras de novas tecnologias e saúde. São Cristovão e Salvador: Editora Universidade de Feira de Santana e Editora da UFBa, pp. 29-56, 2009.

[6] O'HARA, L.; GREGG, J. The war on obesity: A social determinant of health. Health Promotion Journal of Australia. v. 17, n. 3, pp. 260-263, 2006.

[7] ROBISON, J.; PUTNAM, K.; MCKIBBIN, L. Health at every size: A compassionate, effective approach for helping individuals with weight-related concerns - Part I. American Association of Occupational Health Nurses. v. 55, n. 4, pp. 143-150, 2007.

[8] SPAHN, J.M.; et al. State of the evidence regarding behavior change theories and strategies in nutrition counseling to facilitate health and food behavior change. Journal of the American Dietetic Association. v. 110, n. 6, pp. 879891, 2010. 
MARIANA DIMITROV ULIAN doutoranda do Programa de Pós-Graduação em Nutrição em Saúde Pública da Faculdade de Saúde Pública da Universidade de São Paulo (FSP-USP) - e-mail: m.dimitrov@usp.br

FERNANDA BAEZA SCAGLIUSI professora do Departamento de Nutrição da Faculdade de Saúde Pública da Universidade de São Paulo (FSP-USP) - e-mail: fernanda.scagliusi@gmail.com

PRISCILA DE MORAIS SATO doutoranda do Programa de Pós-Graduação Interdisciplinar em Ciências da Saúde da Universidade Federal de São Paulo, campus Baixada Santista e-mail:pri.sato@gmail.com

BRUNO T. MODESTO mestrando da Escola de Educação Física e Esporte da Universidade de São Paulo (EEFE-USP) - e-mail: brunomodesto@usp.br

FABIANA B. BENATTI doutora pela Escola de Educação Física e Esporte da Universidade de São Paulo (EEFE-USP) e pós-doutoranda da Faculdade de Medicina da Universidade de São Paulo (FM-USP) -e-mail:fabenatti@gmail.com

BRUNO GUALANO professor do Departamento de Biodinâmica da Escola de Educação Física e Esporte da Universidade de São Paulo (EEFE-USP) - e-mail: gualano@usp.br

ODILON J. ROBLE professor do Departamento de Educação Física e Humanidades da Faculdade de Educação Física da Universidade Estadual de Campinas - e-mail: roble@fef.unicamp.br

RAMIRO FERNANDEZ UNSAIN professor da Facultad de Filosofía y Letras da Universidad Nacional de Buenos Aires - e-mail: ramirofunsain@yahoo.co.uk 